\title{
Spontaneous synthesis of carbon nanowalls, nanotubes and nanotips using high flux density plasmas
}

\author{
K. Bystrova,*, M. C. M. van de Sanden ${ }^{\mathrm{a}}$, C. Arnas ${ }^{\mathrm{b}}$, L. Marot ${ }^{\mathrm{c}}$, D. Mathys ${ }^{\mathrm{d}}$,

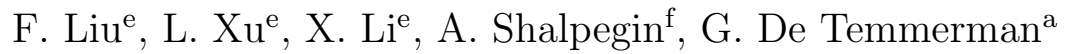 \\ ${ }^{a}$ FOM Institute DIFFER - Dutch Institute for Fundamental Energy Research, \\ Association EURATOM-FOM, Partner in the Trilateral Euregio Cluster, P.O. Box 120\%, \\ NL-3430 BE Nieuwegein, The Netherlands \\ ${ }^{b}$ Laboratoire de Physique des Interactions Ioniques et Moléculaires (PIIM), CNRS, \\ Aix-Marseille Université, F-13397 Marseille, France \\ ${ }^{c}$ Department of Physics, University of Basel, Klingelbergstrasse 82, CH-4056 Basel, \\ Switzerland \\ ${ }^{d}$ Center of Microscopy, University of Basel, Klingelbergstrasse 50/70, CH-4056 Basel, \\ Switzerland \\ e Science and Technology of Marine Corrosion and Protection Laboratory, Luoyang Ship \\ Material Research Institute, 266101, Qingdao, P.R. China \\ ${ }^{f}$ Department of Plasma Physics, National Research Nuclear University MEPhI, 115409, \\ Moscow, Russian Federation
}

\begin{abstract}
We have investigated the formation of various carbon nanostructures using extreme plasma fluxes up to four orders of magnitude larger than in conventional plasma-enhanced chemical vapor deposition processing. Carbon nanowalls, multi-wall nanotubes, spherical nanoparticles and nanotips are among the structures detected with electron microscopy methods. Precursor injection or surface pretreatment were not required for the synthesis of the nanostructures. Preliminary experiments with varied plasma composition, sample bias and surface temperature have demonstrated the potential for optimizing the growth of the nanostructures in the current experimental set-up.
\end{abstract}

*Corresponding author. Tel: +31306096930 , E-mail address: k.bystrov@differ.nl (K. Bystrov) 


\section{Introduction}

The prospect of manipulating and controlling matter on the nanoscale is extremely appealing and offers an enormous opportunity for new technical applications, since artificially produced nanostructures can have useful properties that macroscopic objects don't possess. In recent years, carbon-based nanostructures have drawn a lot of the attention, since the discovery of "buckyballs" in laser-ablated graphite in 1985 and multiwall carbon nanotubes in soot produced by an arc discharge in 1990. A great variety of carbon-based nanostructures has been synthesized since then. Carbon nanofibers[1], nanosheets[2, 3], nanocones[4], nanohorns[5], nanodiamond films[6], nanowalls $[7,8]$ and graphene[9] are just few examples illustrating this variety.

Use of low-temperature plasma systems have become a common way for synthesis of materials with nanoscale features [10]. There are several comprehensive reviews describing plasma-aided nanofabrication, which discuss advances in the field $[11,12]$. It is well known that balance between the rates of material supply and consumption is a critical factor that determines what object is formed on the surface. This makes the precursor species flux and the surface temperature extremely important for grwoth. The use of plasma is advantageous since plasma effects can be used to control the species delivery and heat transfer to the developing nanostrucutres [11]. In other words surface heating can be decreased and the flux increased, both - quite dramatically. This would drive the processes on the surface very far out of equilibrium and will lead to non-equilibrium structures. The higher is the non-equilibrium, the more options arise to produce unusual, self-organizing structures[11]. Theoretical possibility of nanostructure deposition under far-from-equilibrium high flux conditions has been demonstrated numerically, for example for single layer graphene on nickel [13].

Nanostructures are also formed, not always on purpose, in more agressive plasma environments, such as, for example, fusion reactors[14, 15, 16, 17, 18, $19,20,21,22]$ and plasma generators simulating conditions in fusion reactors $[23,24,25]$. While ion energies in fusion-relevant experiments are comparable to those used in various plasma-assisted deposition techniques, the ion and energy fluxes are up to four orders of magnitude larger (Figure 1)[26]. Of 


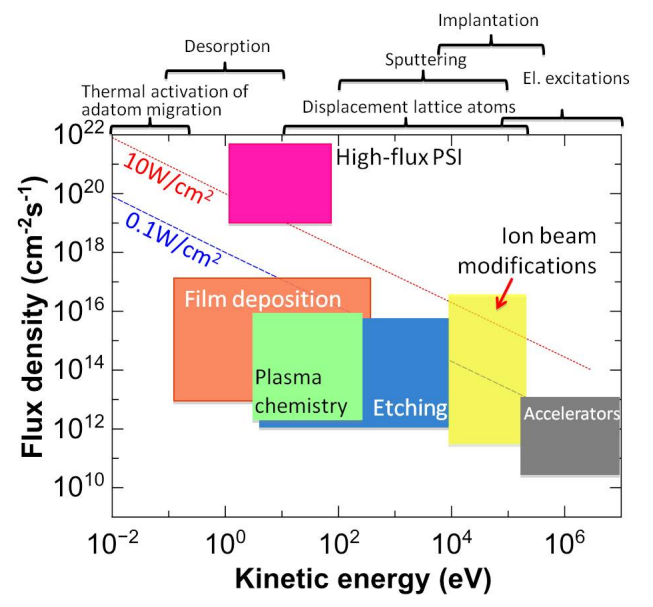

Figure 1: Typical operation windows for various ion-induced processing techniques.

course it remains to be seen whether controlled nanofabrication is in principle possible under such extreme conditions. A curiosity driven question though, is whether a material grown under such extreme conditions would have superior properties, compared to "conventional" materials. Natural diamond is an excellent example demonstrating how harsh conditions during synthesis can promote self-organization. Formed from ordinary carbon atoms in a high pressure, high temperature environment, diamond posesses excellent mechanical and thermal properties. Would then a material synthesized under extreme heat and particle load be resilient to heat and particle bombardment?

Conventionally, low-temperature plasmas with ion densities not exceeding $10^{18} \mathrm{~m}^{-3}$ are used for nanofabrication. Yet, the advantage of increasing carbon influx to the substrate was demonstrated, for example, in experiments with magnetically enhanced arc discharges. It was shown that increase of plasma density and temperature through application of magnetic fields[27, 28] results in increased length of single-walled carbon nanotubes[29] and provides conditions for one-step synthesis of large-scale and high-quality graphene flakes $[28,30]$.

We have performed a series of exploratory experiments at even higher plasma flux densities in order to look for answers to the questions of nanosynthesis under far-from-equilibrium high flux conditions. Experiments were 
done in the linear plasma generator Pilot-PSI, capable of producing plasmas with very high density of up to $10^{21} \mathrm{~m}^{-3}$, with a corresponding ion flux of $\left(\leq 10^{24} \mathrm{~m}^{-2} \mathrm{~s}^{-1}\right)$, and a steady state heat flux of $\leq 10 \mathrm{MWm}^{-2}$. Such heat loads are at the technological limit of what known materials can withstandthey are typical for the exhaust sections of fusion reactors[31] and exceed the loads experienced by a spacecraft during re-entry by at least a factor of ten[32]. Clearly, no additional heating of the sample is required in such set-up. So far, there have been no dedicated investigations of such plasmas as tools for synthesis of carbon nanomaterials, although the possibility for nanostructuring of tungsten and molybdenum surfaces has been already demonstrated [22, 33, 34].

Computer simulations of growth of carbon nanostructures are essential in understanding the underlying physical processes. Recent advances in numercial simulations of growth of carbon nanotubes, as the prototypical example of nanostructured carbon material, have been recently reviewed by Neyts[35] and Elliott[36], who have listed the most advanced methods to date as well as experimental basis the simulations rely on. Molecular dynamics (MD) simulations have become a proven tool fo modelling of carbon nanotube grwoth, also by plasma-assisted methods $[37,38,39,40,41]$. MD simulations of cumulative surface bombardment are very time consuming, which induces a lower limit on the incoming particle flux [42]. Consequently, most of the MD simulations of carbon nanotube growth by plasma-assisted methods employ a carbon addition rate which is more than four orders of magnitude too high[43] compared to a conventional experiment. For quite some time special approaches were needed to include the relaxation effects into the calculations $[44,45,46,47]$, at a price of loosing time information[43]. Recently, Mees[48] proposed a promising forcebias Monte Carlo method with time scale, which enhances the time step compared to classical MD simulations. Still, the problem of extending the limits of the simulated time window to reach different equilibrium states persists $[48,49,50]$. On the other hand, high density plasmas used in this research provide an environment where each surface site is visited every $10 \mu \mathrm{s}$ or less, which is already in the range of typical relaxation times for processes like diffusion of vacancies and interstitials, phase transformation and chemical reactions[26]. Thus, in terms of time scales, such experiments have a potential to become a step towards simulations 


\section{domain.}

\section{Experiment}

Detailed description of the Pilot-PSI plasma generator can be found elsewhere $[51,52,53,54]$. In brief, a cascaded arc source[54, 55] is used to produce the plasma, which exhausts into the vacuum vessel. A strong axial magnetic field confines the plasma, generating an intense magnetized cylindrical plasma beam. Plasma duration in Pilot-PSI is limited by the heating of the magnetic coils. Throughout this work the field of $0.4 \mathrm{~T}$ was used, which allowed plasma exposures of up to $160 \mathrm{~s}$ at a time. It takes approximately 1 $\mathrm{s}$ for the value of the magnetic field to reach its target value in the beginning of a pulse. The time of ramp down of the B-field at the end of the pulse is approximately $0.5 \mathrm{~s}$. Plasma electron density and electron temperature near the plasma-facing surface were measured by Thomson scattering [56].

A water-cooled sample holder is located at a distance of $54 \mathrm{~cm}$ from the exhaust of the plasma source and is installed perpendicularly to the magnetic field. We have used fine-grain graphite samples and polished tungsten samples in these experiments. The samples were discs with the diameter of 30 mm. Prior to exposure all samples were mechanically polished and ultrasonically cleaned using acetone and then alcohol. We used flexible grafoil ${ }^{\circledR}$ sheet as an interface between graphite and the copper heat sink to improve the thermal contact between the two [57]. A clamping ring was shielding a small part of the sample periphery from plasma impact, so the effective exposed diameter of the samples was in fact $26 \mathrm{~mm}$. The samples were negatively biased or electrically floating.

We used argon, hydrogen-argon mixtures and nitrogen as process gases supplied to the inlet of the plasma source. Gas throughput in these experiments ranged from 1.4 to $3.0 \mathrm{slm}$ (standard liter per minute; $1 \mathrm{slm}=4.4$ $\times 10^{20}$ particles/s). Additionally, in experiments with metal substrates we have used methane injection as a source of carbon. Methane was injected through a ring surrounding the plasma column and located several centimeters downstream from the source exit. Injection took place only during the magnetic field pulses with the injection rate of $0.25 \mathrm{slm}$. The schematic of the experiment is shown in Figure 2.

A fast infrared (IR) camera (SC7500-MB, FLIR) was employed to monitor the surface temperature of the samples during exposures. The waveband of the camera spans from $1.5 \mu \mathrm{m}$ to $5.1 \mu \mathrm{m}$. 2D surface temperature 


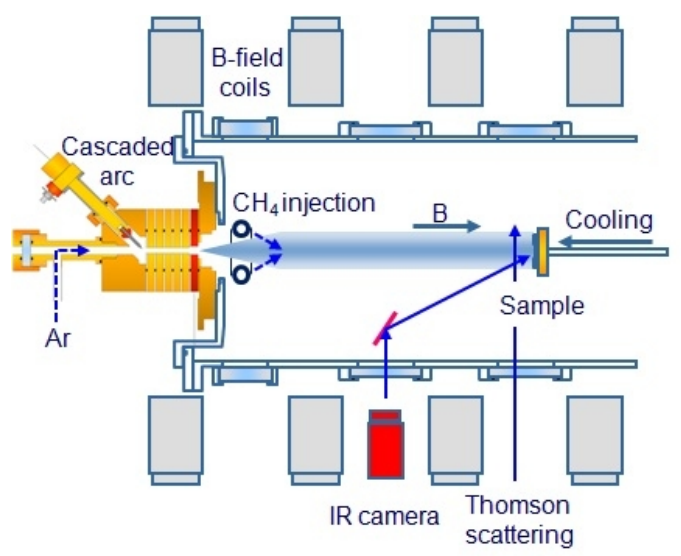

Figure 2: Schematic view of the deposition experiment in the Pilot-PSI linear plasma generator. Methane is injected into an argon plasma beam through a ring situated near the nozzle of the plasma source.

profiles were measured with a spatial resolution of $0.3 \mathrm{~mm}$. Scanning electron microscopy (SEM) and high-resolution transmission electron microscopy (HRTEM), were used to image the formed nanostructures. X-ray photoelectron spectroscopy (XPS, PHI Quantera SXM ${ }^{T M}$, ULVAC-PHI, Inc.) was used to analyze the chemical properties of the modified surfaces. Selected samples were analyzed with visible Raman spectroscopy and X-ray diffraction (XRD).

\section{Results}

\subsection{Growth of nanostructures on tungsten with precursor gas injection}

In order to synthesize carbon structures on tungsten, we have exposed a tungsten sample to argon plasma with methane injection. Mixtures of argon and methane are used for conventional plasma-enhanced chemical vapor deposition (PECVD) processing [12, 58], while the main feature of this experiment is the high ion flux density from the plasma. The "high flux PECVD" exposure was done in two pulses for a total duraction of 100 seconds. The sample was electrically floating. The plasma near the sample had an electron temperature of $1.1 \mathrm{eV}$ and an electron density of $1.0 \times 10^{20} \mathrm{~m}^{-3}$. This corresponds to a flux density of approximately $2 \times 10^{23} \mathrm{~m}^{-2} \mathrm{~s}^{-1}$ in the middle of the beam and a fluence of around $1 \times 10^{25} \mathrm{~m}^{-2}$, assuming that argon is the dominant ion in the plasma. 
During the exposure the entire plasma exposed area turned completely black, as tungsten was entirely covered by deposits. The XPS spectrum in Figure 3a shows that carbon is the main element on the surface, followed by oxygen. Oxygen was most likely adsorbed during the post-plasma exposure of the sample to air. Note, that no tungsten is detected with XPS. The C 1s core level spectrum is plotted in Figure 3b. The fitting curves indicate a multicomponent structure. The most intensive component corresponds to carbon in a zero oxidation state in the $\mathrm{sp}^{2}(284.6 \mathrm{eV})$ and $\mathrm{sp}^{3}(285.1 \mathrm{eV})$ hybridization states. The ratio between the $\mathrm{sp}^{2}$ and $\mathrm{sp}^{3}$ states is approximately 2-to1. Such ratio is close to what is found for $\mathbf{a}-\mathrm{C}: \mathbf{H}$ films of various hardness $[59,60]$. A minor peak at $289.2 \mathrm{eV}$ appears due to $-\mathrm{COOH}$ groups usually observed for carbon materials. It should be noted that no indications of carbide formation were found in the XPS spectrum, likely due to the thickness of the deposits.

The Raman spectrum of the produced nanostructured deposits is shown in Figure 4. The spectrum has a strong peak at $1590 \mathrm{~cm}^{-1}$ ( $\mathrm{G}$ band) and another peak at $1347 \mathrm{~cm}^{-1}$ (D band). According to Hiramatsu and Hori [61] the $\mathrm{G}$ band indicates the formation of graphitized structure, while the $\mathrm{D}$ band corresponds to the disorder-induced phonon mode. The $\mathrm{G}$ band peak is accompanied by a shoulder peak at $1620 \mathrm{~cm}^{-1}$. This peak is often designated as $\mathrm{D}^{\prime}$ peak and associated with finite-size graphite crystals and graphene edges [61]. The strong D peak and the the shoulder peak are typical for Raman spectra of carbon nanowalls, which is due to the small graphite domains and existence of edges of graphene sheets. The relative peak intensity $I_{D} / I_{G}$ in the spectrum is approximately 2, while the width of the $\mathrm{G}$ band is about $50 \mathrm{~cm}^{-1}$.

Electron microscopy provided insight into the morphology of the carbon deposits. Overview SEM image shows that the substrate is covered by carbon particles as shown in Figure 5a. An SEM image with higher magnification (Figure 5b) indicates that each particle has nanostructured surface with detectable carbon nanowall-like features. The TEM images of deposits, scratched from the surface (Figures 5c,d) show graphite structures composed of crumpled graphene layers. These graphene layers have typical lengths of several tens of nanometers and thicknesses of around $5 \mathrm{~nm}$.

Electron microscopy images point to the fact that the surfaces of microparticles consist of crumpled carbon nanowalls (CNW). Indeed, the deposited structures consist of graphite sheets and have edges composed of stacked graphene, consistent with the definition of CNW by Hiramatsu and 

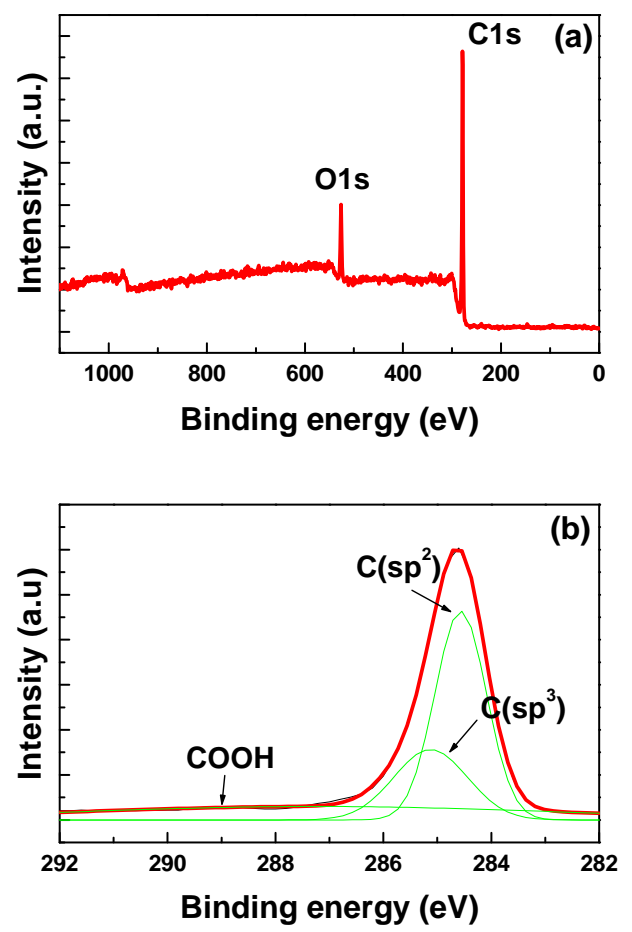

Figure 3: Results of the XPS analysis of deposit formed on a tungsten substrate using argon plasma with methane injection. The overview spectrum (a) shows that the film consists of carbon. Oxygen was most likely adsorbed during post-plasma exposure to air. Multi-component structure of the $\mathrm{C}$ 1s line (b) indicates the presence of $\mathrm{sp}^{2}$ and $\mathrm{sp}^{3}$ hybridization states. 


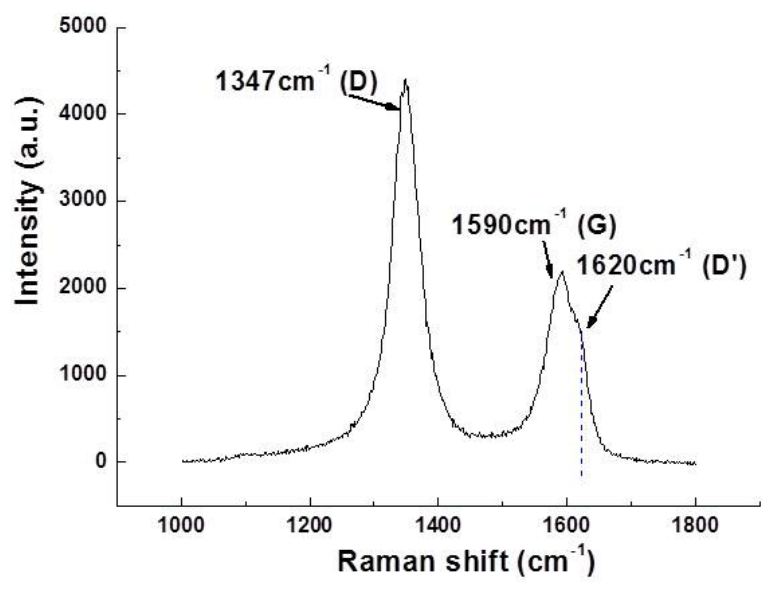

Figure 4: Raman spectrum of nanostructured deposits produced using argon plasma with methane injection. The $\mathrm{G}$ band indicates the formation of graphitized structure, while the $\mathrm{D}$ band together with a smaller D band is due to the presence of graphite disorder.
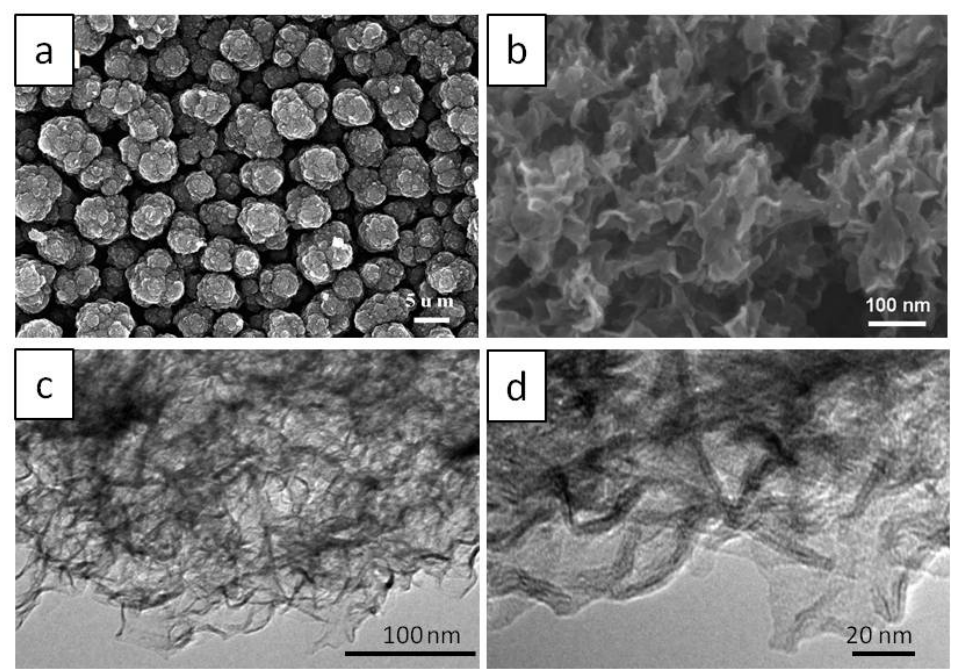

Figure 5: Electron microscopy images of carbon structures synthesized on a tungsten substrate using argon plasma with methane injection. SEM images (a,b) show carbon microparticles covering the substrate. The microparticles have structured surfaces with carbon nanowall-like features. These nanowalls which are composed of stacked graphene layers are seen on the TEM images $(\mathrm{c}, \mathrm{d})$. 


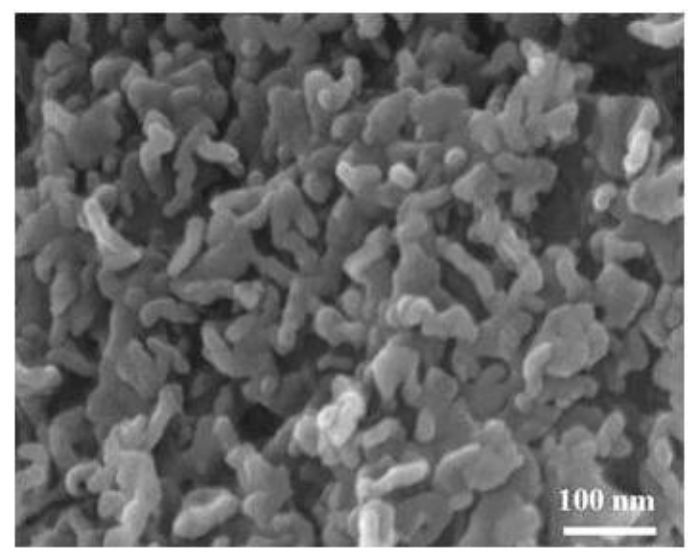

Figure 6: SEM image showing entangled cylindrical nanostructures formed in the region of the peak particle and heat flux.

Hori [61]. Nanowalls synthesized in Pilot-PSI are however more crumpled than the textbook examples of CNW[61]. This is consistent with a high level of disorder and explains the features of the Raman spectrum characteristic of disordered graphite, namely the $I_{D} / I_{G}$ ratio of approximately 2 , and rather narrow $G$ and $D$ bands with respect to what is usually found for amorphous carbon. The $I_{D} / I_{G}$ ratio in our spectrum is relatively low and the $\mathrm{G}$ band is wide in comparison with the typical spectra for CNW[62].

Another part of the definition of carbon nanowalls postulates that they should stand almost vertically on the substrate [61]. In our case, however, the original substrate is buried beneath the layer of carbon microparticles. Experiments on CNW growth using glow discharges[63] demonstrated that the formation of such spherical particles is in fact one of the grwoth stages. The spherical particles were observed at longer durations and higher ion fluxes compared to those needed for the "classical" CNW film synthesis. It is plausible that the particle formation is a result of coagulation and agglomeration induced by further development of previously grown graphene sheets [63]. Such an explation fits well with the magnitudes of ion fluxes in our experiments.

The microscopic structure of the spherical particles depends on their location with respect to the center of the sample. Close to the center of the sample the surfaces of the particles consist of entangled cylindrical nanostructures (Figure 6). The differences in morphology between the center and the edge of the sample could have been caused by surface temperature gradient. 


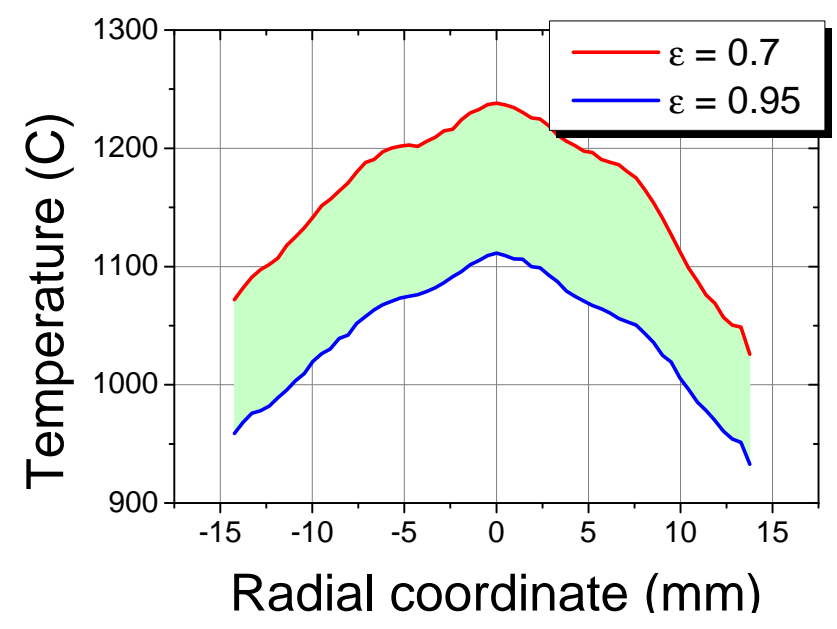

Figure 7: Steady state surface temperature during deposition of carbon micro- and nanostructures. Since the surface emissivity coefficient for the deposits is unknown, the expected minimal and maximal temperatures at any given point are estimated using tabulated values of emissivity coefficients for various carbonaceous materials.

Higher temperatures in the center of the sample could have caused rolling-up of the carbon layers. Such behaviour was observed with hydrogenated amorphous carbon layers in PECVD experiments, where the temperature increase produced a partial hydrogen desorption and, consequently, re-organization of the carbon bonds [64].

Temperature measurements for this sample were not trivial and require some explanation. Results of infrared (IR) thermography measurements during the methane injection experiment are plotted in Figure 7 . The figure shows a radial distribution of temperature after 60 seconds of exposure, when tungsten is already covered by carbon. Intrepretation of the IR data relies on the value of spectral emissivity of the surface, which is a function of wavelength and depends on the surface morphology. In our case, it is difficult to assign a concrete value of emissivity to the surface, since the properties of the deposits have not yet been studied in details and may have changed during the exposure. We have used typical tabulated values for the surface emissivity coefficient for carbonaceous materials, which range from 0.70 for graphite to 0.95 for carbon soot, to rougly estimate the upper and the lower limits of the temperature in any given point. The actual temperature value is between the two extremes (shadowed area in Figure 7). One additional comment is 
that during previous experiments with graphite the surface emissivity in the center of the sample has been determined using a multiwavelength pyrometer. It turned out that the value of around 0.95 was needed to match the IR data with the pyrometer measurements. The cental regions of exposed graphite samples and the film deposited here look quite similar, both being covered by deposited carbon microparticles, as will be mentioned further. It is then likely that at least in the center of the sample the emissivity was close to 0.95. In any case, the temperature gradient across the sample is created by the Gaussian shape and the width of the plasma beam. Indeed, the plasma beam is narrower than the diameter of the samples.

Considering the above, we assume that the temperature in the center of the sample was close to $1100{ }^{\circ} \mathrm{C}$, while the periphery was somewhat colder between $970{ }^{\circ} \mathrm{C}$ and $1050{ }^{\circ} \mathrm{C}$. Here it is necessary to recall that because of the high heat flux (of the order of $10 \mathrm{MWm}^{-2}$ ), the sample heating happens entirely due to plasma exposure and that no external heating is required.

Finally, the substrate with deposits was analyzed with XRD (Figure 8). Comparison of XRD spectra for a pristine and exposed samples allows us to conclude that tungsten carbide has in fact formed during the plasma treatment. It is not surprising, since formation of an interface carbide layer is the usual initial stage of carbon film growth on metal substrates $[65,66]$. Carbide layer formation was then followed by formation of nanostructured carbon deposits. As the thickness of deposited material increased, the incoming ions no longer interacted with the original tungsten substrate. In other words, the carbide layer was buried under the layers of deposited carbon and protected from the plasma.

A question whether the tungsten substrate played a catalytic role during the growth of the described deposits remains open. At this moment there is no evidence neither in favor, nor against this statement. However, we do know that similar nanostructured carbon particles can be deposited on various substrates - such as graphite (Section 3.2 of this paper) and molybdenum[67]. So, even if tungsten was playing a catalytic role in these experiments, it is clearly not required to perform the deposition of the carbon particles. 


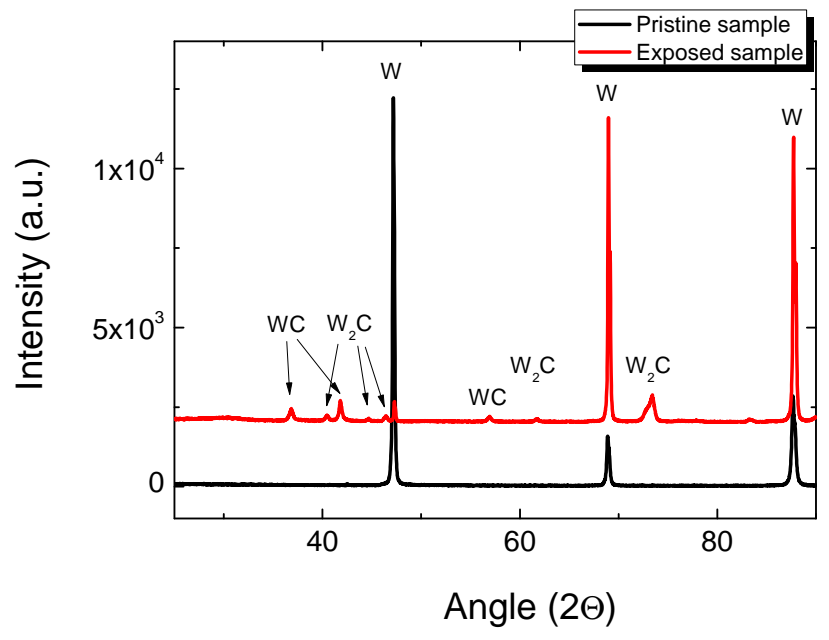

Figure 8: XRD spectra for a pristine tungsten sample and a sample with deposited carbon structures. Extra peaks on the latter spectrum correspond to tungsten carbide formed during initial stages of deposition.

\subsection{Growth of nanostructures on graphite without precursor gas injection}

\subsubsection{Nanostructured carbon microparticles}

The previous section describes carbon nanostructures formed on tungsten during exposure to high flux plasma. In that experiment carbon as building material for the nanostructures has been supplied via an upstream injection of methane. Methane molecules were dissociated and ionized by the intense argon plasma beam while carbon, hydrocarbon and hydrogen ions were created in the process. However, external methane (or, in fact, any other precursor gas) injection is not required to synthesize carbon nanostructures in Pilot-PSI. The substrate itself can be a source of precursors for the synthesis of carbon nanostructures.

Chemical sputtering of graphite samples by intense hydrogen plasma beams leads to quick formation of carbon microparticles in the region of the highest plasma flux as we have demonstrated earlier[25]. The deposits are formed because the plasma is so dense that it is not transparent for hydrocarbon molecules that are chemically sputtered from graphite. Very strong recycling of carbon leads to re-organization of the plasma-exposed surface and appearance of the microparticles. To avoid ambiguity it is necessary to specify that by chemical sputtering we understand "a 
process whereby ion bombardment causes or allows a chemical reaction to occur, which produces a particle that is weakly bound to the surface and hence can be easily desorbed into the gas phase" $[68,69]$. Chemical sputtering yield depends on both the kinetic energy and the chemical reactivity of the impinging species [69].

The structure of the microparticles have been further investigated with SEM and TEM (Figures 9 and 10, respectively). It is obvious that the particles are similar to those deposited on tungsten with methane injection or particles consisting of carbon nanowalls grown in a much more quiescent environment of PECVD discharges $[63,70,71]$. In this case we again find the features characteristic for carbon nanowalls in the material forming the microparticles. For instance graphene layers marking the edges of the nanowalls are shown in TEM images in Figure 9. The number of atomic carbon layers in pictured graphenes varies in the range from 5 to 10.

Experiments with varied deposition conditions are required to gain understanding of the carbon structures growth. We have started investigating the effect of deposition conditions on the surface morphology by varying argon concentration in the plasma and the sample bias. Plasmas with $33 \%$ and $57 \%$ of argon (in terms of gas flow) were produced by varying the relative flows of gases supplied to the inlet of the plasma source. Two samples were exposed for a duration of 210 seconds, with the plasma parameters being $T_{e}$ $=0.7 \mathrm{eV}$ and $n_{e}=2.0 \times 10^{20} \mathrm{~m}^{-3}$. A reference sample was exposed to pure hydrogen plasma with similar parameters. The samples have been negatively biased at $-30 \mathrm{~V}$ to accelerate the ions from the plasma. The peak surface temperature for all samples was $1100{ }^{\circ} \mathrm{C} \pm 100{ }^{\circ} \mathrm{C}$.

SEM images of the synthesized carbon structures for both samples and the reference case with no argon in the plasma are shown in Figure 10. All SEM images depict structures formed in the center of the samples. The sample exposed to the mixture with $57 \%$ of argon has longer nanowalls, separated by larger distances. The estimated length of CNW in this case reaches several hundred nanometers. Following the classification of CNW suggested by Hiramatsu and Hori[61] we consider these structures to be wavy nanowalls. For the case with $33 \%$ of argon the nanowalls are shorter and less ordered, just like in the reference scenario with pure hydrogen. In principle, dilution of hydrocarbon-containing plasmas with argon is an established method for preventing excessive amorphous carbon deposition during PECVD synthesis of carbon nanostructures $[12,58]$. It is possible that at lower argon flows carbon deposition was happening too fast, masking the 

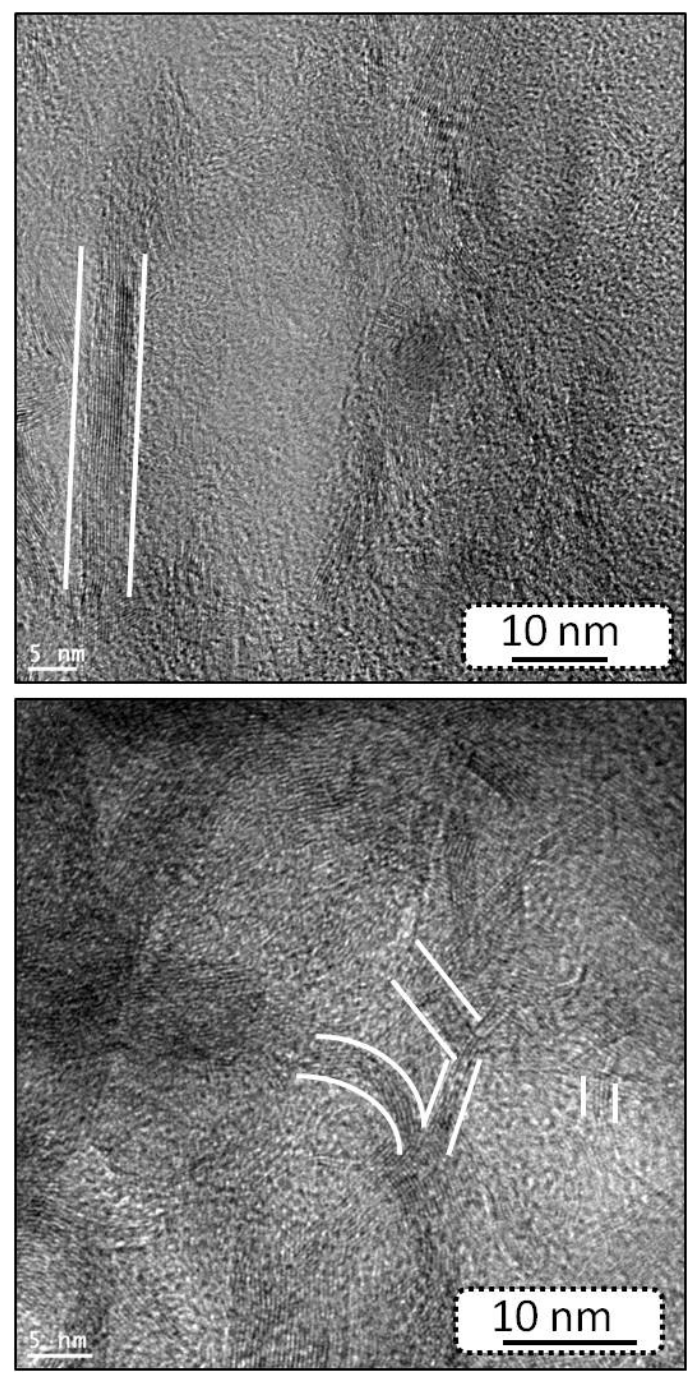

Figure 9: TEM images of the internal structure of carbon microparticles deposited on graphite without precursor gas injection. 


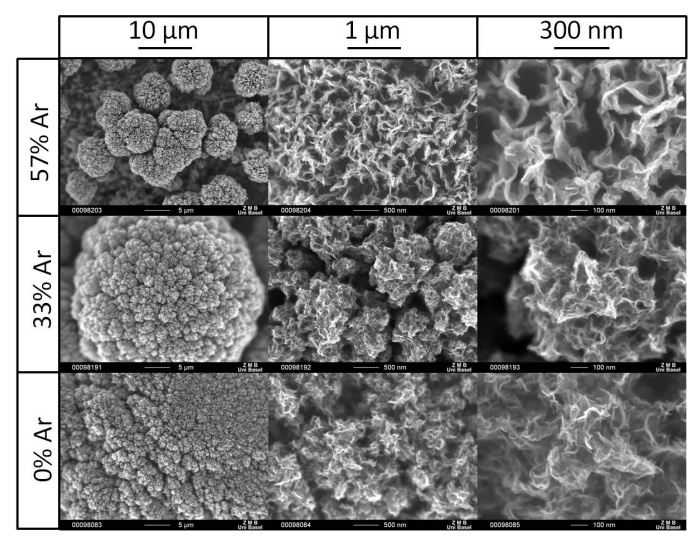

Figure 10: SEM images showing the impact of argon admixture into the plasma on the structure of carbon microparticles deposited on graphite without precursor gas injection. Addition of argon is beneficial for the growth of larger carbon nanowalls. All samples were biased at $-30 \mathrm{~V}$.

nanowalls.

Increase of the absolute value of the bias voltage has a similar effect on the surface as addition of $57 \%$ of argon. Figure 11 shows an SEM image of a surface exposed to pure hydrogen, but with the bias voltage of $-50 \mathrm{~V}$, instead of $-30 \mathrm{~V}$ used for the samples described above. Energetic hydrogen ions would sputter amorphous carbon faster compared to graphite [72, 73, 74], effectively aiding synthesis of more organized structures. On a microscopic level the morphology remains the same in all cases - centers of all samples are covered by carbon particles.

Apart from carbon nanowalls, spherical nanoparticles and carbon nanotips are found in materials redeposited on the same surfaces. The examples are shown in Figure 12. Again, there are examples of very similar nanostructures formed using PECVD processing techniques[75, 76]. Hassouni [77] described how spherical carbon particles can grow in argon discharges with methane injection (and hydrogen for discharge stabilization). The growth is volumetric and the basic units for the growth are polycyclic aromatic hydrocarbon molecules. Spherical carbon nanoparticles that we find in PilotPSI[25] also point towards volumeteric growth, although the exact reactions responsible for it have not yet been identified. It is intriguing that the surface temperature in our experiments, and probably also the neutral gas temperature in the immediate vicinity of the surfaces, is close to the optimal gas temperature for nucleation in the model describtion of dusty microwave dis- 


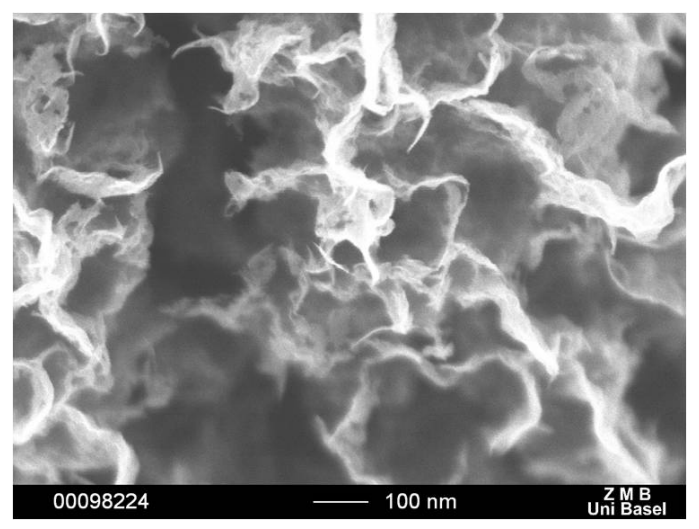

Figure 11: SEM image of carbon nanowalls formed on graphite exposed to pure hydrogen plasma. The sample was biased at $-50 \mathrm{~V}$.

charges [77].

\subsubsection{Multi-wall carbon nanotubes and carbon nanotips}

Another example of the synthesis of carbon nanostructures by high density plasma is the formation of carbon nanotubes during exposure of graphite to nitrogen plasma. The nanotubes were also synthesized on graphite substrates without injection of precursor gas. Nitrogen was used as a working gas in the cascaded arc source. The plasma temperature and density were $T_{e}$ $=0.9 \mathrm{eV}$ and $n_{e}=1.5 \times 10^{20} \mathrm{~m}^{-3}$ in this experiment. The sample was biased at $-30 \mathrm{~V}$ and exposed for $300 \mathrm{~s}$ up to a fluence of around $1 \times 10^{26} \mathrm{~N}^{+} \mathrm{m}^{-2}$. The temperature in the middle of the sample was around $900{ }^{\circ} \mathrm{C}$ according to the IR camera measurement.

The nanotubes were first noticed during SEM surveys of the exposed sample. The overview image of the modified graphite surface is shown in Figure 13a. The surface coverage by the nanotubes is not homogeneous, instead, they are clustered in selected places. Images with higher magnification as well as HRTEM analysis revealed that the structures are multi-wall carbon nanotubes (Figure 13b,c). The nanotubes lengths are typically less than $500 \mathrm{~nm}$ and their diameters are between $5 \mathrm{~nm}$ and $10 \mathrm{~nm}$. Judging by their lengths, the growth rate of the nanotubes was of the order of $1 \mathrm{~nm} / \mathrm{s}$. The nanotubes in this exploratory experiment are bended which is usually related to defect creation in the catbon lattice [78, 79].

Apart from the nanotubes there are chains of nanoparticles on the surface. A high resolution SEM image of such nanoparticle chains is shown in 


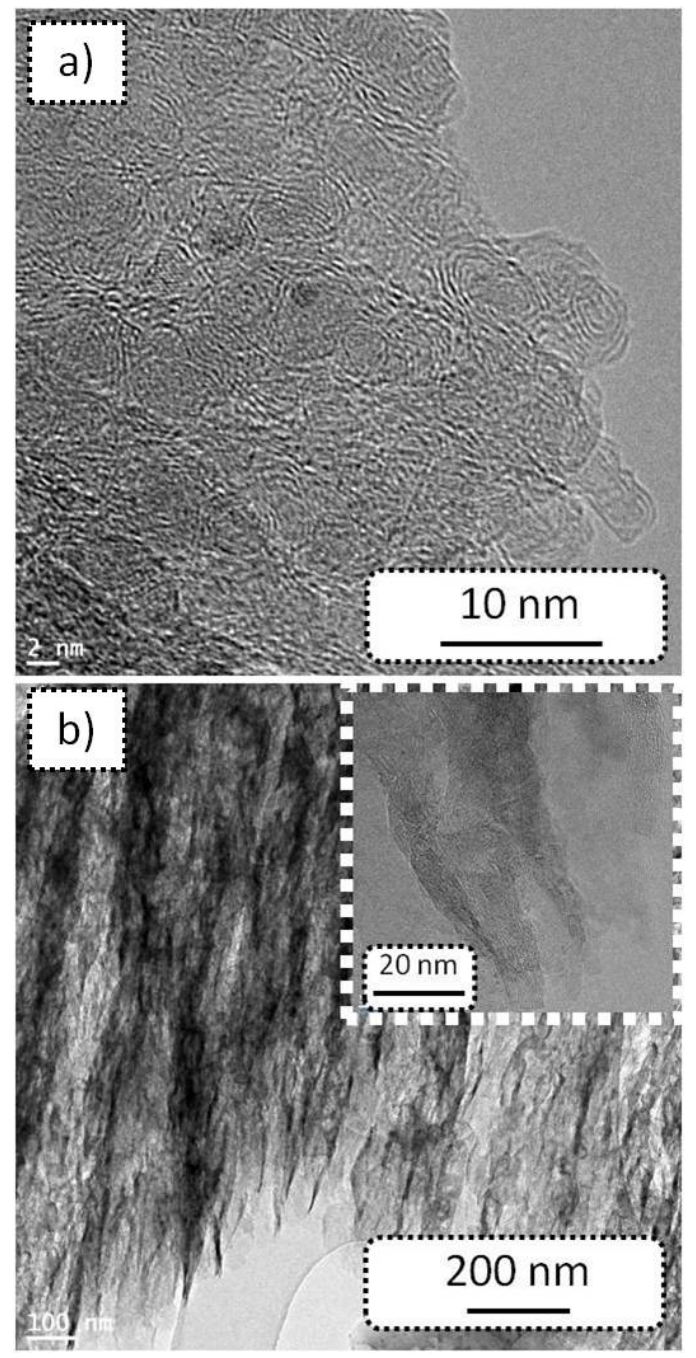

Figure 12: Spherical nanoparticles (a) and carbon nanotips (b) are among nanostructures found in the deposits formed on graphite without precursor gas injection. 

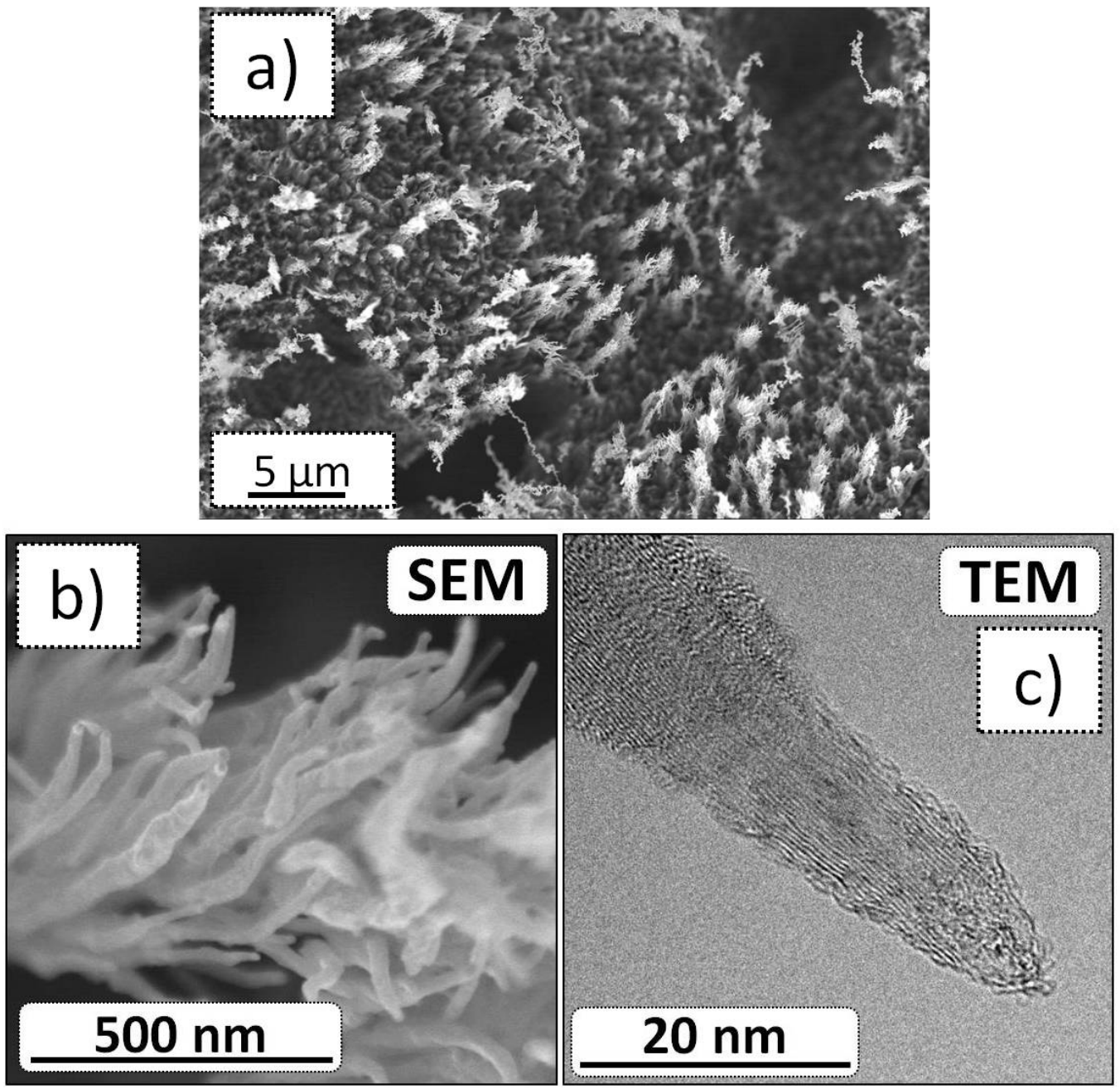

Figure 13: SEM image of the graphite surface modified by high flux nitrogen plasma beam. Multiple clusters of carbon nanotubes and chains of nanoparticles protrude from the roughened surface(a). SEM and HRTEM images of multi-wall carbon nanotubes synthesized on graphite using nitrogen plasma without precursor gas injection $(b, c)$. 
Figure 14a. The typical size of the nanoparticles in a chain is around 100 nm. The length-to-thickness ratio of a chain of nanoparticles can be rather high. For example, the longest chain on the images we have obtained was about 5 microns, while it is less than $20 \mathrm{~nm}$ thick in narrower places. TEM observations suggest that the particles are metallic and the XPS spectrum (Figure 15) implies that the particles are either molybdenum or lanthanum. The atomic concentrations of molybdenum and lanthanum on the surface were found to be $4.2 \%$ and $0.3 \%$, respectively. Molybdenum originates from the sputtering of the clamping ring surrounding the sample, while lanthanum is a dopant used in the tungsten cathodes of the plasma source. The presence of these metals on the surface is probably caused by the mass and reactivity of nitrogen ions, since we do not observe them in hydrogen or argon plasmas. The presence of oxygen and its bonding with carbon and nitrogen makes it difficult to determine whether $\mathrm{C}-\mathrm{N}$ bonds have formed during the plasma exposure.

In some places the carbon nanotubes are attached to the chains of the nanoparticles (Figure 14b,c). It is known that molybdenum [80] and lanthanum [81] are catalysts for nanotube growth and as such could have played a crucial role in this experiment. Figure 14b,c suggests that the nanotubes were growing on top of the metal nanoparticles. At the same time, analysis of the TEM images shows that there are no metallic particles on the tips of the nanotubes. It is worth noting that we have not observed the nanotubes on electrically floating samples or on samples exposed to significantly lower fluxes of nitrogen[82], when in both cases neither molybdenum, nor lanthanum were present in the system.

Increase of the exposure time to $1020 \mathrm{~s}$ resulted in increased concentration of metallic nanoparticles on the surface. Moreover, after 1020 seconds carbon nanostructures do not cluster, but instead cover the entire surface (Figure 16a). The synthesized structures are carbon nanotips (CNTP)[76] in this case. The original graphite surface is very rough, with many microscopic hills and pits. These features remain on the surface after exposure and their sidewalls are also covered by the nanotips, implying that the angle at which a given surface is positioned with respect to the plasma does not play a big role for their synthesis. It is interesting to note the orientation of the nanotipsthey are all perpendicular to the surface. A close-up image of the nanotips shows that they are rather thick, probably covered by amorphous carbon on their sides (see Figure 16b). Once again, it is explainable, since no measures were taken to remove the amorphous phase and optimize the growth. Yet, 


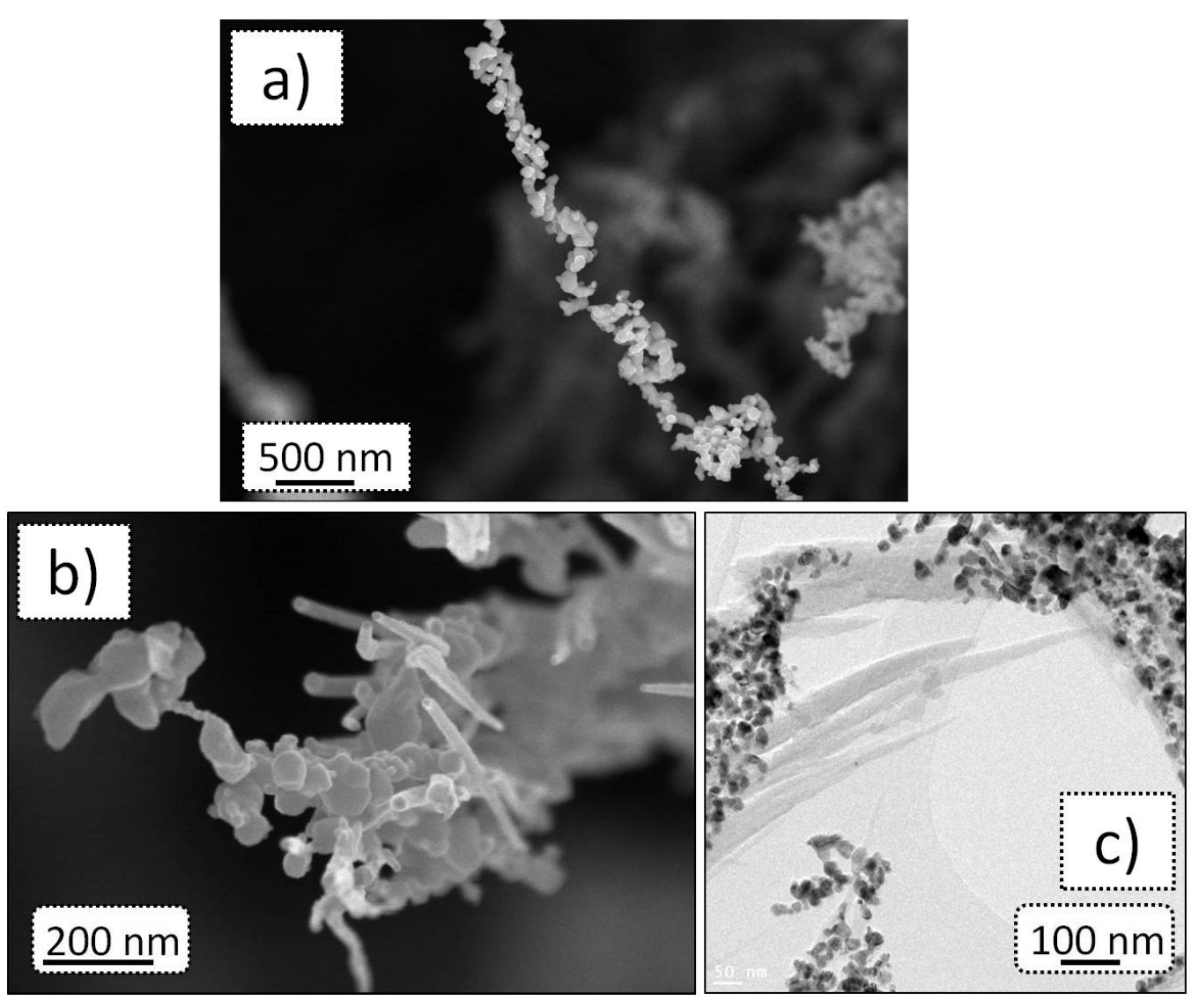

Figure 14: (a)SEM image of a nanochain composed of metallic nanoparticles. Such nanochains are formed during exposure of graphite to high flux nitrogen plasma in presense of small amounts of molybdenum and lanthanum. SEM (b) and TEM (c) images of carbon nanotubes attached to metallic nanoparticles. 


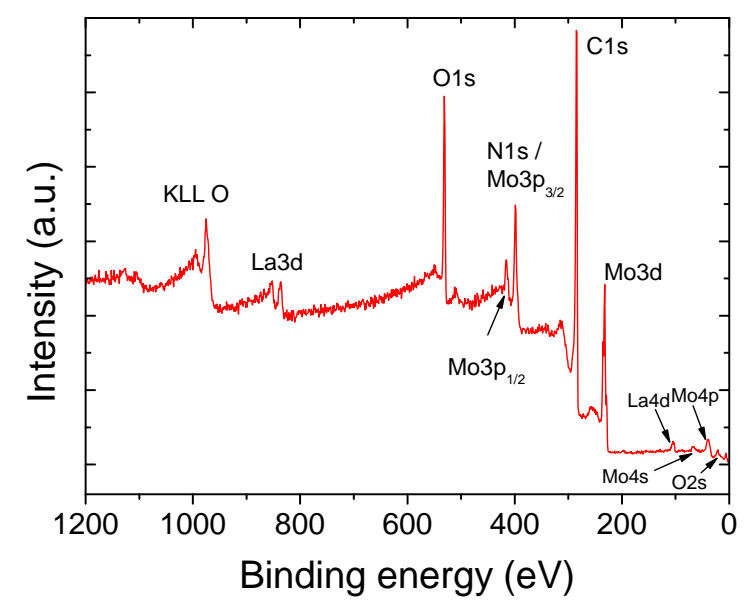

Figure 15: An overview XPS spectrum of the graphite sample exposed to high flux nitrogen plasma.

as Figure 16c indicates, the ends of the nanotips can be graphitic. In this experiment, the metal nanoparticles are not only organized in chains, many of them are capping the carbon nanotips (Figure 16b) or are attached to their sides (Figure 16c).

We have varied the surface temperature during the exposure to see if it plays a role for the CNTP synthesis. The effect shown in Figure 17 is quite clear: CNTP synthesized at a temperature of $1200{ }^{\circ} \mathrm{C}$ are very thick and have a tendency to adhere to their neighbours, while nanotips grown at a relatively low surface temperature of $660{ }^{\circ} \mathrm{C}$ are significantly thinner and the thickness is uniform along the length. Such differences could be explained in terms of temperature dependence of the chemical sputtering yield of carbon by nitrogen. As a reminder, we note that by chemical sputtering we understand a process of removal of weakly bound chemical compounds that were formed during bombardment by energetic ions from the surface. We have shown that the chemical sputtering yield of carbon by nitrogen peaks at around $670{ }^{\circ} \mathrm{C}$ and decreases at higher temperatures in Pilot-PSI[82]. So, at the temperature of $660{ }^{\circ} \mathrm{C}$ the unnecessary carbon deposits would be more readily sputtered away, enabling formation of thin long carbon nanostructures on the surface. 

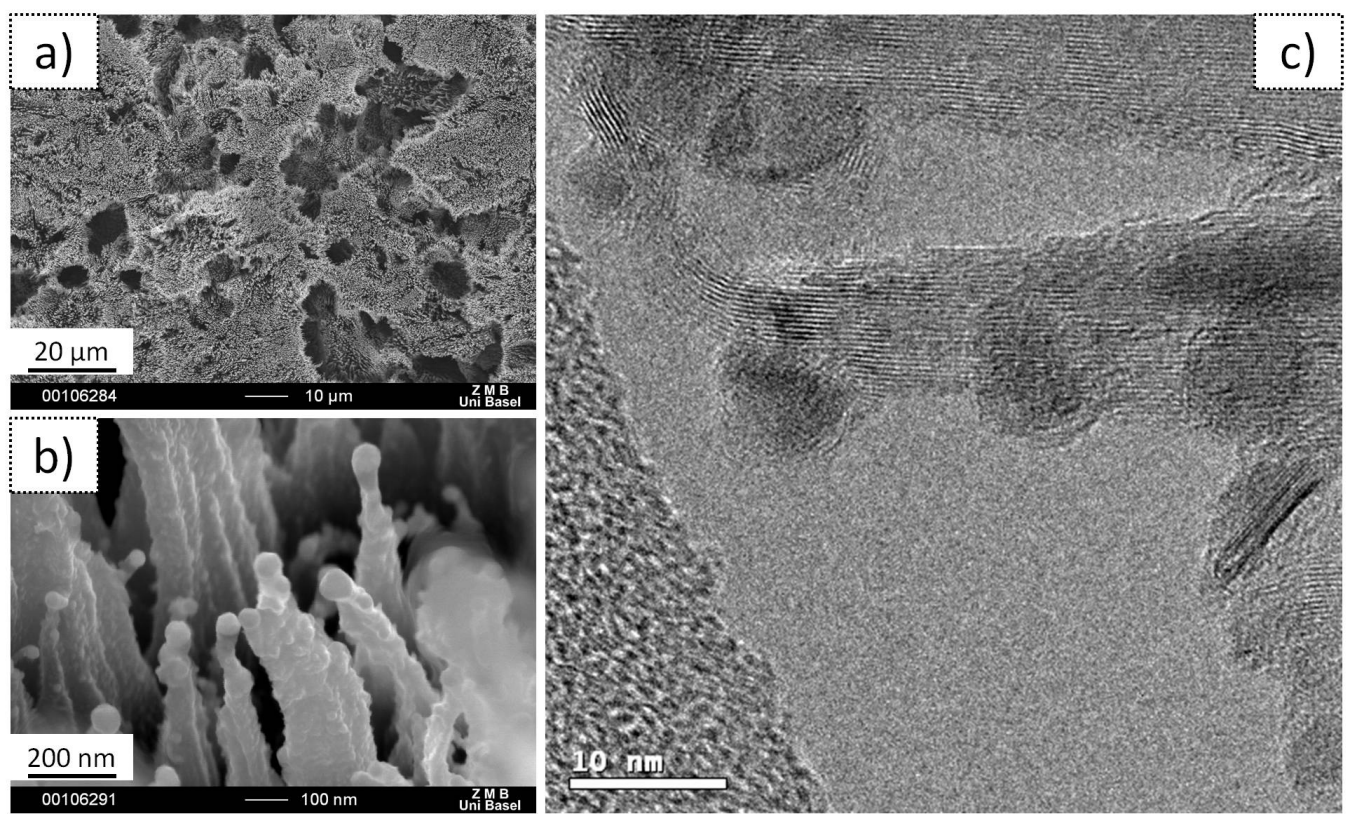

Figure 16: SEM image of carbon nanotips covering the entire graphite surface after 17 minutes of exposure to high flux nitrogen plasma without precursor gas injection (a). Close-up image of the carbon nanotips show that metallic nanoparticles can cap them (b). HRTEM image of a carbon nanotip decorated with metal nanoparticles (c). 


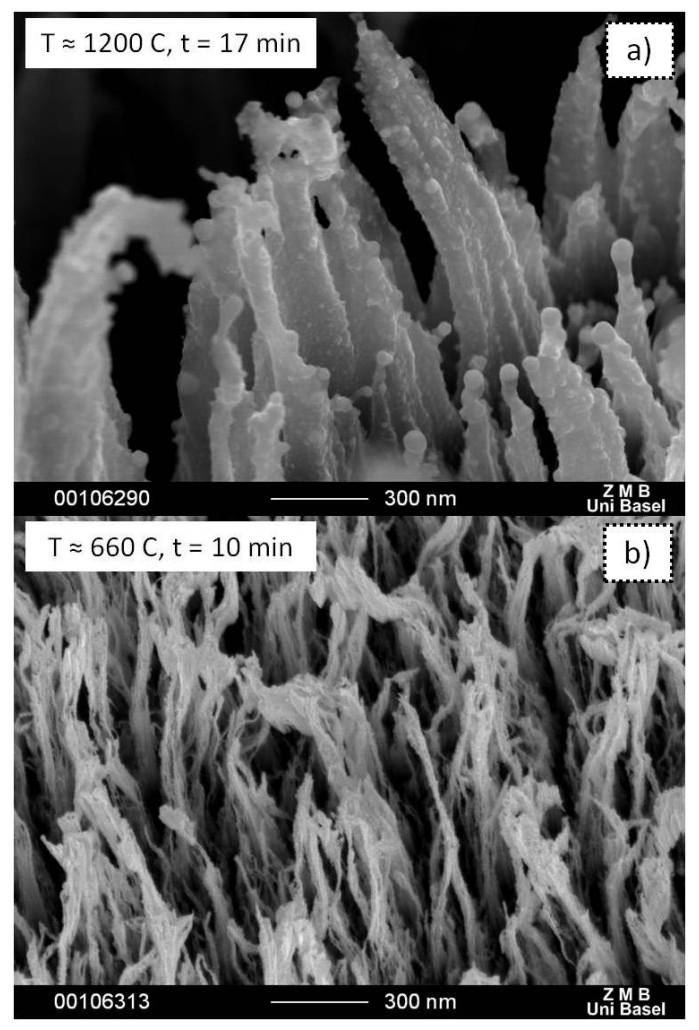

Figure 17: The effect of surface temperature on the growth of carbon nanotips. Nanotips grown at high temeperature are very thick and seem to accrete with their neighbours (a), while lower temperatures promote growth of significantly thinner nanotips (b). 


\section{Discussion and outlook}

We have presented a number of findings about carbon nanostructures formed in a high density plasma environment. These experiments had an exploratory purpose and we tried to demonstrate the variety of nanostructures that can be formed under extreme plasma bombardment. Below we discuss the possible ways of optimizing the deposition conditions by controlling carbon migration in such experiments.

The carbon deposition rate (and, consequently, the growth rate of carbon microparticles) depends on the concentration of hydrocarbons in the plasma. It is relatively easy to vary the hydrocarbons concentration when precursor gas is injected externally. The problem becomes more complicated when carbon is supplied by the substrate itself via sputtering, as is the case in a number of experiments described here. We have determined that recycling of carbon can be very strong (up to 90\%) under high flux plasma bombardment[25]. This actually implies that controlling the growth of the nanostructures in such environment is not possible without controlling the carbon migration, including sputtering, transport and re-deposition. It is well known that the chemical sputtering yield by hydrogen depends strongly on the surface temperature[69]. One could think of varying the heat flux, sample and cooling geometry in order to control the amount of carbon being sputtered from the sample. Carbon migration through the plasma and redeposition under described plasma loading conditions are being extensively studied with the 3D Monte Carlo impurity transport and plasma-surface interactions code ERO [83, 84]. Ultimately, one could think of using such code as a predictive model for deposition experiments.

One additional note is that particles with similar nanofeatures appeared on a tungsten substrate in methane injection experiments and on graphite samples in experiments without injection. Moreover, similar deposits have also been observed on molybdenum substrates [67], meaning that the substrate does not play a critical role in their synthesis.

The case of nanotube growth during exposures of graphite to nitrogen might not be that different from the deposition of nanostructured microparticles in hydrogen and hydrogen-argon atmosphere. Nitrogen chemically sputters carbon atoms in the form of volatile molecules[85, 86, 87, 88, 89, 90, 91, $92,93]$ that can then be trapped by the dense plasma beam. Similarly to the case of microparticle formation, the plasma-exposed graphite surface is a source of carbon for nanostructure formation. It is still unclear how large 
is the redeposition coefficient in nitrogen, with surface profilometry suggesting that it is less pronounced compared to the case of hydrogen[82]. This is probably why it was relatively easy to detect the nanotubes, since not that much carbon was redeposited around them.

It is necessary to study the effect of metallic particles on the growth of nanotubes. The first step would be to determine which nanostructures, if any, would be formed by the nitrogen plasma beam in a metal-free environment. It is possible to eliminate molybdenum by using a graphite clamping ring. Sputtering of lanthanum from the cathodes of the plasma source can be prevented if nitrogen is injected into the beam close to the nozzle of the plasma source, instead of into the cathode chamber. The second step would be to expose graphite samples doped with molydbenum, lanthanum and other potential catalysts in order to separate the effects of various metals on the nanostrucuture formation.

To summarize, there is a number of ways to optimize the deposition conditions in a linear generator of high flux density plasmas. Such experiments could provide input to the existing growth models of the nanostructures, and would lead to the development of the deposition techniques using far-fromequilibrium high flux conditions.

\section{Conclusions}

We have demonstrated that a variety of carbon nanostructures can be synthesized using high density low temperature plasmas with flux densitites exceeding $10^{23} \mathrm{~m}^{-2} \mathrm{~s}^{-1}$. Carbon nanowalls, nanoparticles, multi-wall nanotubes and nanotips are among the structures that were detected with electron microscopy. The synthesis experiments are summarized in Table 1.

Injection of methane into hydrogen plasma leads to carbon deposition on a tungsten substrate. The deposit is in the form of carbon microparticles with recognizable carbon nanowall-like features on their surface. Apart from nanowalls, spherical nanoparticles and nanotips are found by transmission electron microscopy. Carbon particles with similar nanofeatures are found on graphite surfaces exposed to hydrogen and hydrogen-argon plasmas with similar parameters.

Neither precursor gas injection, nor special substrate pre-treatment were required to synthesize the nanostructures on graphite. The substrates themselves serve as sources of hydrocarbon molecules which are chemically sputtered by hydrogen ions coming from the plasma. Strong recirculation of 


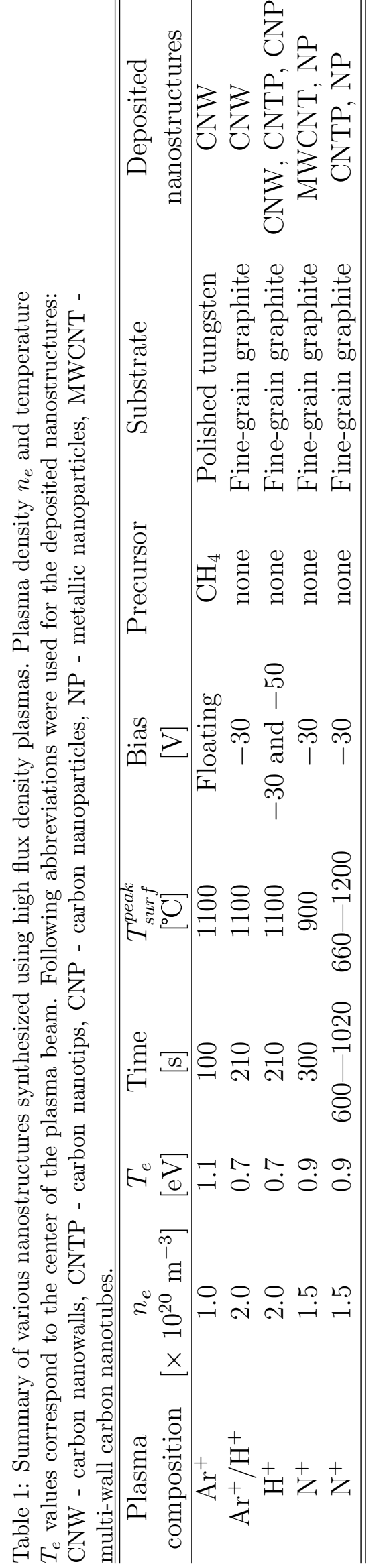


carbon is responsible for the fact that many sputtered particles return to the surface and form the deposits. Multiwall carbon nanotubes and nanotips are formed on graphite surfaces exposed to high fluxes of nitrogen, also without precursor injection and surface pretreatment. The nanotubes and nanotips were formed in the presence of trace amounts of molydbenum and lanthanum that could have played a role of catalysts. The metals are present on the surface in the form of nanoparticles, which can form micrometer-long chains. Some nanoparticles are attached to the nanotips.

The synthesis of the nanostructures happened spontaneously in a sense that plasma and surface conditions during growth were not optimized in any way. This fact explains the sometimes excessive level of disorder in the deposits. We have nevertheless observed that admixture of argon into the plasma leads to more structured nanowall patterns on the surface of the microparticles. Similar effect was observed on the sample exposed to ions with higher energy. Variation of surface temperature influenced the thickness of the carbon nanotips formed in nitrogen. These examples demonstrate the potential of controlling the growth of the nanostructures in the current experimental set-up. Careful variation of such experimental conditions as surface temperature, ion energy, and plasma impurity content, as well as extensive analysis of deposits would be necessary to understand the formation of each type of the nanostructures and optimize their synthesis.

These experiments provide an interesting insight into the behaviour of materials under extreme particle and heat fluxes. Synthesis of carbon nanostructures is an intriguing and somewhat surprizing result. It is necessary to seek understanding and control of the mechanisms that guide the formation of the nanostructures. Controlled manipulation of materials in extreme non-equilibrium environments may lead to discoveries of structures with superior properties, not obtainable by conventional techniques.

\section{Acknowledgements}

The authors would like to thank Richard Al, Marc van de Pol, Hennie van der Meiden, Peter Wortman and the rest of the Pilot-PSI team for their support in preparation and execution of the experiments. This work was supported by the European Communities under the Contract of Association between EURATOM/FOM and carried out within the framework of the European Fusion Programme with financial support from NWO and the NWO Grant RFBR 047.018.002. This work was also made within the framework of EFDA and a contract of the French FR FCM - EURATOM/CEA association. The 
views and opinions expressed herein do not necessarily reflect those of the European Commission. 


\section{References}

[1] M. Endo and H. W. Kroto. J. Phys. Chem., 96:6941, 1992.

[2] Q. Kuang, S.-Y. Xie, Z.-Y. Jiang, X.-H. Zhang, Z.-X. Xie, R.-B. Huang. Carbon, 42:1737, 2004.

[3] J. Wang, M. Zhu, R. A. Outlaw, X. Zhao, D. M. Manos, and B. C. Holloway. Carbon, 42:2867, 2004.

[4] K. Sattler. Carbon, 33:915, 1995.

[5] S. Iijima, M. Yudasaka, R. Yamada, S. Badrow, K. Suenaga, F. Kokai, et al. Chem. Phys. Lett., 309:165, 1999.

[6] L. Qin, D. Zhou, A. R. Krauss, and D. M. Gruen. Nanostructured Materials, 10:649, 1998.

[7] Y. Ando, X. Zhao and M. Ohkohchi. Carbon, 35:153, 1997.

[8] Y. Wu, P. Qiao, T. Chong, and Z. Shen. Advanced Materials, 14:64, 2002.

[9] A. K. Geim and K. S. Novoselov. Nature Materials, 6:183, 2007.

[10] K. Ostrikov and S. Xu. Plasma-Aided Nanofabrication. Wiley-VCH, 2007.

[11] K. Ostrikov, E. C. Neyts, and M. Meyyappan. Adv. Phys., 62:113, 2013.

[12] M. Meyyappan, L. Delzeit, A. Cassell, and D. Hash. Plasma Sources Sci. Tech., 12:205, 2003.

[13] E. C. Neyts, A. C. T. van Duin, and A. Bogaerts. Nanoscale, 5:7250, 2013.

[14] B. N. Kolbasov, A. B. Kukushkin, V. A. Rantsev-Kartinov, and P. V. Romanov. Phys. Lett. A, 269:363, 2000.

[15] B. N. Kolbasov, A. B. Kukushkin, V. A. Rantsev-Kartinov, and P. V. Romanov. Phys. Lett. A, 291:447, 2001. 
[16] M. Richou, C. Martin, P. Delhaes, M. Couzi, W. Saikaly, C. Brosset, et al. Carbon, 45:2723, 2007.

[17] P. Roubin, B. Pégourié, R. Smirnov, C. Martin, M. Richou, Y. Marandet, et al. J. Nucl. Mater., 390-391:49, 2009.

[18] C. Arnas, C. Pardanaud, C. Martin, P. Roubin, G. De Temmerman, and G. Counsell. J. Nucl. Mater., 401:130, 2010.

[19] C. Arnas, C. Martin, P. Roubin, B. Pégourié, G. De Temmerman, K. Hassouni, et al. Plasma Phys. Contr. Fusion, 52:124007, 2010.

[20] V. I. Krauz, Yu. V. Martynenko, N. Yu. Svechnikov, V. P. Smirnov, V. G. Stankevich, and L. N. Khimchenko. Physics - Uspekhi, 53:1015, 2010 .

[21] N. Asakura, T. Hayashi, N. Ashikawa, T. Hatae, and T. Nakano. Fusion Sci. Technol., 60:1572, 2011.

[22] G. M. Wright, D. Brunner, M. J. Baldwin, K. Bystrov, R. P. Doerner, B. LaBombard, et al. J. Nucl. Mater., 438:S84, 2013.

[23] N. Ohno, Y. Kobayashi, T. Sugimoto, and S. Takamura. J. Nucl. Mater., $337: 35,2005$.

[24] N. Ohno, M. Yoshimi, M. Tokitani, S. Takamura, K. Tokunaga, and N. Yoshida. J. Nucl. Mater., 390-391:61, 2009.

[25] K. Bystrov, L. van der Vegt, G. De Temmerman, C. Arnas, and L. Marot. J. Vac. Sci. Technol. A, 31:011303, 2013.

[26] G. De Temmerman, et al. Acta Polytechnica, 53:142, 2013.

[27] M. Keidar, A. M. Waas, Y. Raitses, and E. Waldorff. J. Nanosci. Nanotechnol., 6:1309, 2006.

[28] I. Levchenko, O. Volotskova, A. Shashurin, Y. Raitses, K. Ostrikov, and M. Keidar. Carbon, 48:4556, 2010.

[29] M. Keidar, I. Levchenko, T. Arbel, M. Alexander, A. M. Waas, and K. Ostrikov. Appl. Phys. Lett., 92:043129, 2008. 
[30] O. Volotskova, I. Levchenko, A. Shashurin, Y. Raitses, K. Ostrikov, and M. Keidar. Nanoscale, 2:2281, 2010.

[31] A. Herrmann, T. Eich, S. Jachmich, M. Laux, P. Andrew, A. Bergmann, et al. J. Nucl. Mater., 313-316:759, 2003.

[32] E. A. Meese and H. Nørstrud. Aerosp. Sci. Technol., 6:185, 2002.

[33] G. De Temmerman, K. Bystrov, J. J. Zielinski, M. Balden, G. Matern, C. Arnas, et al. J. Vac. Sci. Technol. A., 30:041306, 2012.

[34] G. De Temmerman, K. Bystrov, R. P. Doerner, L. Marot, G. M. Wright, K. B. Woller, et al. J. Nucl. Mater., 438:S78, 2013.

[35] E. C. Neyts. J. Vac. Sci. Techno. B, 30:030803, 2013.

[36] J. A. Elliott, Y. Shibuta, H. Amara, C. Bichara, and E. C. Neyts. Nanoscale, 5:6662, 2013.

[37] S. Irle, Y. Ohta, Y. Okamoto, A. J. Page, Y. Wang, and K. Morokuma. Nano Research, 2:755, 2009.

[38] Y. Shibuta. Diamond Relat. Mater., 20:334, 2011.

[39] M. Shariat, S. I. Hosseini, B. Shokri, and E. C. Neyts. Carbon, 65:269, 2013.

[40] E. C. Neyts, K. Ostrikov, Z. J. Ham, S. Kumar, A. C. T. van Duin, and A. Bogaerts. Phys. Rev. Lett., 110:065501, 2013.

[41] E. C. Neyts, A. C. T. van Duin, and A. Bogaerts. J. Am. Chem. Soc., 134:1256, 2012.

[42] E. D. de Rooij, U. von Toussaint, A. W. Kleyn, and W. J. Goedheer. Phys. Chem. Chem. Phys., 11:9823, 2009.

[43] E. C. Neyts, Y. Shibuta, A. C. T. van Duin, and A. Bogaerts. ACS Nano, 4:6665, 2010.

[44] G. Dereli. Mol. Simul., 8:351, 1992.

[45] M. Timonova, J. Groenewegen and B. J. Thijsse. Phys. Rev. B, 81:144107, 2010. 
[46] E. C. Neyts and A. Bogaerts. Phys. Chem. C, 113:2771, 2009.

[47] E. C. Neyts, A. C. T. van Duin, and A. Bogaerts. J. Am. Chem. Soc., 133:17225, 2011.

[48] M. J. Mees, G. Pourtois, E. C. Neyts, B. J. Thijsse, and A. Stesmans. Phys. Rev. B, 85:134301, 2012.

[49] A. F. Voter, F. Montalenti, and T. C. Germann. Ann. Rev. Mater. Res., $32: 321,2002$.

[50] D. Perez, B. P. Uberuaga, Y. Shim, J. G. Amar, and A. F. Voter. Annu. Rep. Comp. Chem., 5:79, 2009.

[51] V. P. Veremiyenko. Ph.D. thesis, An ITER-relevant magnetized hydrogen plasma jet. Eindhoven, The Netherlands, 2012.

[52] J. Westerhout, W. R. Koppers, W. A. J. Vijvers, R. S. Al, S. Brezinsek, S. Brons, et al. Phys. Scr., T128:18, 2007.

[53] G. J. van Rooij, V. P. Veremiyenko, W. J. Goedheer, B. de Groot, A. W. Kleyn, P. H. M. Smeets, et al. Appl. Phys. Lett., 90:121501, 2007.

[54] W. A. J. Vijvers, C. A. J. van Gils, W. J. Goedheer, H. J. van der Meiden, D. C. Schram, V. P. Veremiyenko, et al. Phys. Plasmas, 15:093507, 2008 .

[55] G. M. W. Kroesen, D. C. Schram, and J. C. M. de Haas. Plasma Chem. Plasma P., 10:531, 1990.

[56] H. J. van der Meiden, R. S. Al, C. J. Barth, A. J. H. Donné, R. Engeln, W. J. Goedheer, et al. Rev. Sci. Instrum., 79:013505, 2008.

[57] M. A. van den Berg, S. Brons, O. G. Kruijt, J. Scholten, R. Pasquet, P. H. M. Smeets, et al. Fus. Eng. Design, 86:1745, 2011.

[58] L. Delzeit, I. McAninch, B. A. Cruden, D. Hash, B. Chen, J. Han et al. J. Appl. Phys., 91:6027, 2002.

[59] J. Robertson. Mater. Sci. Eng. R., 37:129, 2002.

[60] P. Koidl, C. Wagner, B. Dischler, J. Wagner, and M. Ramsteiner. Mater. Sci. Forum, 52:41, 1990. 
[61] M. Hiramatsu and M. Hori. Carbon Nanowalls. Synthesis and Emerging Applications. Springer-Verlag, 2010.

[62] S. Kurita, A. Yoshimura, H. Kawamoto, T. Uchida, K. Kojima, M. Tachibana, et al. J. Appl. Phys., 97:104320, 2005.

[63] Z. Bo, K. Yu, G. Lu, P. Wang, S. Mao, and J. Chen. Carbon, 49:1849, 2011.

[64] I. Géraud-Grenier, V. Massereau-Guilbaud and A. Plain. Surf. and Coat. Technol., 187:336, 2004.

[65] P. Reinke and P. Oelhafen. Diam. Relat. Mater., 8:155, 1999.

[66] P. Reinke and P. Oelhafen. Surf. Sci., 468:203, 2000.

[67] K. Bystrov, L. B. van der Vegt, G. A. van Swaaij, T. Zaharia, Y. Kuang, W.J. Goedheer, et al. J. Nucl. Mater., 438:S686, 2013.

[68] H. F. Winters and J. W. Coburn. Surf. Sci. Rep., 14:162, 1992.

[69] W. Jacob and J. Roth. Chemical Sputtering. In R. Behrisch, W. Eckstein, editor, Sputtering by Particle Bombardment, pages 329-400. Springer, Berlin Heidelberg New York, 2007.

[70] Z. Bo, Y. Yang, J. Chen, K. Yu, J. Yan, and K. Cen. Nanoscale, 5:5180, 2013.

[71] A. T. H. Chuang, B. O. Boskovic, and J. Robertson. Diam. Relat. Mater., 15:1103, 2006.

[72] E. Vietzke, K. Flaskamp, V. Philipps, G. Esser, P. Wienhold, and J. Winter. J. Nucl. Mater., 145-147:443, 1987.

[73] D. L. Youchison, M. D. Nahemow, R. T. McGrath, and A. J. Baratta. J. Nucl. Mater., 176-177:461, 1990.

[74] K. Katayama, H. Nagase, C. Nishinakamura, T. Takeishi, and M. Nishikawa. Fus. Eng. Design, 81A:247, 2006.

[75] N. Aggadi, C. Arnas, F. Bénédic, C. Dominique, X. Duten, F. Silva, et al. Diamond Relat. Mater., 15:908, 2006. 
[76] Z. L. Tsakadze, K. Ostrikov, and S. Xu. Surf. Coat. Technol., 191/1:49, 2005.

[77] K. Hassouni, F. Mohasseb, F. Bénédic, G. Lombardi, and A. Gicquel. Pure Appl. Chem., 78:1127, 2006.

[78] S. Iijima, T. Ichihashi, and Y. Ando. Nature, 356:776, 1992.

[79] S. H. Lim, H. S. Yoon, J. H. Moon, K. C. Park, and J. Jang. Appl. Phys. Lett., 88:033114, 2006.

[80] D. Yuan, L. Ding, H. Chu, Y. Feng, T. P. McNicholas, and J. Liu. Nano Letters, 8:2576, 2008.

[81] Y. An, Q. Hou, J. Wang, Zh. Zheng, H. Zhao, and G. Zhang. J. Rare Earth., 28:717, 2010.

[82] K. Bystrov, T. W. Morgan, I. Tanyeli, G. De Temmerman, and M. C. M. van de Sanden. J. Appl. Phys., 114:133301, 2013.

[83] A. Kirschner, V. Philipps, J. Winter and U. Kögler. Nucl. Fusion, 40:989, 2000.

[84] G. A. van Swaaij, K. Bystrov, D. Borodin, A. Kirschner, T. Zaharia, L. B. van der Vegt, et al. J. Nucl. Mater., 438:S629, 2013.

[85] W. Jacob, C. Hopf and M. Schlüter. Appl. Phys. Lett., 86:204103, 2005.

[86] N. V. Novikov, M. A. Voronkin, A. A. Smekhnov, N. I. Zaika, and A. P. Zakharchuk. Diam. Relat. Mater. , 4:390, 1995.

[87] P. Hammer and W. Gissler. Diam. Relat. Mater., 5:1152, 1996.

[88] R. Kaltofen, T. Sebald, and G. Weise. Thin Solid Films, 290-291:112, 1996.

[89] J. Hong and G. Turban. Diamond Relat. Mater., 8:572, 1999.

[90] S. Grigull, R. Behrisch, and S. Parascandola. J. Nucl. Mater., 275:158, 1999.

[91] N. A. Morisson, S. E. Rodil, J. Robertson, and W. I. Milne. J. Appl. Phys., 89:5754, 2001. 
[92] N. Hellgren, M.P. Johansson, E. Broitman, P. Sandström, L. Hultman, and J.-E. Sundgren. Thin Solid Films, 382:146, 2001.

[93] Z. W. Deng and R. Souda. Surf. Sci., 488:393, 2001. 


\section{Figure captions}

Fig. 1. Typical operation windows for various ion-induced processing techniques.

Fig. 2. Schematic view of the deposition experiment in the Pilot-PSI linear plasma generator. Methane is injected into an argon plasma beam through a ring situated near the nozzle of the plasma source.

Fig. 3. Results of the XPS analysis of deposit formed on a tungsten substrate using argon plasma with methane injection. The overview spectrum (a) shows that the film consists of carbon. Oxygen was most likely adsorbed during post-plasma exposure to air. Multi-component structure of the $\mathrm{C} 1 \mathrm{~s}$ line (b) indicates the presence of $\mathrm{sp}^{2}$ and $\mathrm{sp}^{3}$ hybridization states.

Fig. 4. Electron microscopy images of carbon structures synthesized on a tungsten substrate using argon plasma with methane injection. SEM images (a,b) show carbon microparticles covering the substrate. The microparticles have structured surfaces with carbon nanowall-like features. These nanowalls which are composed of stacked graphene layers are seen on the TEM images $(\mathrm{c}, \mathrm{d})$.

Fig. 5. Raman spectrum of nanostructured deposits produced using argon plasma with methane injection. The $\mathrm{G}$ band indicates the formation of graphitized structure, while the $\mathrm{D}$ band together with a smaller D band is due to the presence of graphite disorder.

Fig. 6. SEM image showing entangled cylindrical nanostructures formed in the region of the peak particle and heat flux.

Fig. 7. Steady state surface temperature during deposition of carbon micro- and nanostructures. Since the surface emissivity coefficient for the deposits is unknown, the expected minimal and maximal temperatures at any given point are estimated using tabulated values of emissivity coefficients for various carbonaceous materials.

Fig. 8. XRD spectra for a pristine tungsten sample and a sample with deposited carbon structures. Extra peaks on the latter spectrum correspond to tungsten carbide formed during initial stages of deposition.

Fig. 9. TEM images of the internal structure of carbon microparticles deposited on graphite without precursor gas injection.

Fig. 10. SEM images showing the impact of argon admixture into the plasma on the structure of carbon microparticles deposited on graphite with-

out precursor gas injection. Addition of argon is beneficial for the growth of larger carbon nanowalls. All samples were biased at $-30 \mathrm{~V}$.

Fig. 11. SEM image of carbon nanowalls formed on graphite exposed to 
pure hydrogen plasma. The sample was biased at $-50 \mathrm{~V}$.

Fig. 12. Spherical nanoparticles (a) and carbon nanotips (b) are among nanostructures found in the deposits formed on graphite without precursor gas injection.

Fig. 13. SEM image of the graphite surface modified by high flux nitrogen plasma beam. Multiple clusters of carbon nanotubes and chains of nanoparticles protrude from the roughened surface.

Fig. 14. SEM image of the graphite surface modified by high flux nitrogen plasma beam. Multiple clusters of carbon nanotubes and chains of nanoparticles protrude from the roughened surface(a). SEM and HRTEM images of multi-wall carbon nanotubes synthesized on graphite using nitrogen plasma without precursor gas injection $(\mathrm{b}, \mathrm{c})$.

Fig. 15. An overview XPS spectrum of the graphite sample exposed to high flux nitrogen plasma.

Fig. 16. SEM image of carbon nanotips covering the entire graphite surface after 17 minutes of exposure to high flux nitrogen plasma without precursor gas injection (a). Close-up image of the carbon nanotips show that metallic nanoparticles can cap them (b). HRTEM image of a carbon nanotip decorated with metal nanoparticles (c).

Fig. 17. The effect of surface temperature on the growth of carbon nanotips. Nanotips grown at high temeperature are very thick and seem to accrete with their neighbours (a), while lower temperatures promote growth of significantly thinner nanotips (b). 